\title{
ANNE-AËL ROPARS
}

\section{Université de Brest}

\section{Les Soixante-quinze Feuillets de Marcel Proust ou la fabrique du masque}

« $\begin{aligned} & \text { otre figure ne change guère : un doigt de } \\ & \text { poudre, il n'y paraît presque pas, car notre }\end{aligned}$ figure c'est notre moi, et en somme on nous reconnaît toujours " ${ }^{1}$, affirme le narrateur de Jean Santeuil à propos de Perrotin, personnage atteint d'un cancer et cachant sa maladie derrière une apparence mondaine. Proust est lucide : le masque ne fait pas illusion. Lui qui, dans Le Temps retrouvé, démasquera les personnalités du fameux «bal des têtes », confirmant par là qu' « on nous reconnaît toujours »malgré le passage du temps et le travestissement, essaye pourtant, dès 1895, de se cacher derrière son personnage de Jean Santeuil - un échec, comme l'écrit Jérôme Picon, car «la pluralité des voix et des sources de récit, comme l'extension du point de vue extérieur au personnage principal, cause du "masque" déguisant à peine l'auteur, ne convenaient guère à créer le "visage littéraire" qui forme le foyer principal du grand-œuvre proustien ${ }^{2}$.

1 M. Proust, Jean Santeuil, Paris, Gallimard, 2001, p. 681.

2 M. Proust, Correspondance, J. Picon (éd. critique), Paris, 
En effet, si l'on reconnaît Proust derrière le narrateur d'À la recherche du temps perdu, son "visage littéraire » est une construction romanesque autrement plus complexe que dans sa première mouture. De l'un à l'autre, le parcours du narrateur est émaillé de brouillons, ébauches et entreprises littéraires dont chaque étape révèle les clefs du masque proustien. En ce sens, la parution récente des Soixante-quinze Feuillets et autres manuscrits inédits ${ }^{3}$ de Marcel Proust apporte une clef essentielle. II s'agit de la plus ancienne version de La Recherche, composée de six manuscrits (soixante-seize feuillets exactement), que les proustiens croyaient disparus depuis leur évocation par Bernard de Fallois en $19544^{4}$. D'une valeur génétique immense, Les Soixante-quinze Feuillets datent probablement de la fin de l'année 1907 ou du début de l'année 1908 ; soit huit ans après la rédaction interrompue de Jean Santeuil

Flammarion, 2007, p. 14. Le « visage littéraire » est une expression de J.-Y. Tadié tirée de Proust et le roman, Paris, Gallimard, 1971, p. 22.

3 M. Proust, Les Soixante-quinze Feuillets et autres manuscrits inédits, N. Mauriac-Dyer (éd. critique), Paris, Gallimard, 2021. Les citations suivantes provenant de cette œuvre seront marquées à l'aide de l'abréviation SF (pagination après le signe abréviatif).

4 M. Proust, Contre Sainte-Beuve, B. de Fallois (éd. critique), Paris, Gallimard, 1954, p. 14. 
(1895-1899), roman autobiographique dans lequel Proust se cache maladroitement derrière une narration à la troisième personne, et cinq ans avant la publication du premier volume de La Recherche où il assume la première personne tout en niant le caractère autobiographique du roman.

Les Soixante-quinze Feuillets offrent donc un matériau nouveau et antérieur aux Cahiers, brouillons de La Recherche à partir de 1908. Dans ces manuscrits, la superposition de deux univers - réel et romanesque - laisse encore largement apparaître l'inspiration autobiographique du récit, tout en annonçant très nettement l'imaginaire de la Recherche. En témoignent les thèmes abordés, qui donnent leur titre aux six fragments: «Une soirée à la campagne ", "Le côté de Villebon et le côté de Meséglise » (avant-textes du Côté de chez Swann), "Séjour au bord de la mer », "Jeunes filles » (avant-textes des Jeunes filles en fleurs), « Noms nobles » et "Venise », deux rêveries qui traverseront La Recherche et sont déjà en germe dans Les Soixante-quinze Feuillets. Nous assistons ici à la genèse de la création romanesque, à ses revirements, à ses contradictions; Proust transforme sa réalité en lui appliquant un ou plusieurs masques - pseudonymes, fusion de personnes réelles dans un même personnage ou, au contraire, éclatement d'une personne réelle dans plusieurs personnages, 
superposition d'espaces géographiques : nous voici pleinement dans le laboratoire de l'écriture et, par conséquent, dans la fabrique du masque, ces « masques de littérature » dont le narrateur parle dans Jean Santeuil ${ }^{5}$ à propos de Claude Le Roux, son professeur de philosophie qui se fait appeler Clodius Rustinlor, pseudonyme transparent et prétentieux dont se moque le narrateur. Proust, lui, va élaborer un art du pseudonyme bien plus riche, comme l'annoncent déjà Les Soixantequinze Feuillets.

Le masque comme protection de la vie privée

Dans un premier temps, le masque apparaît sous la plume de Proust comme une astuce pour protéger sa vie privée et celle de son entourage. Comme l'écrit Jean-Yves Tadié dans sa Préface à Jean Santeuil : "Proust censure ce que sa mère ne peut pas lire » ${ }^{6}$. En 1908, Jeanne Weil est décédée depuis trois ans, mais Proust cède encore à ce subterfuge censé déjouer l'investigation des biographes - on sait Proust sensible à cette problématique puisqu'il en fera l'année suivante l'objet de son essai contre Sainte-Beuve où il prône la dissociation de l'œuvre et de la vie de l'écrivain.

5 M. Proust, Jean Santeuil, op. cit., p. 109.

6 Ibidem, p. 8. 
Dans Les Soixante-quinze Feuillets, le masque se matérialise d'abord comme une absence, celle de thématiques qui prendront une importance majeure dans La Recherche : le judaïsme et I'homosexualité, sujets que Proust n'osera aborder qu'à travers les fameux « masques de littérature ». Rappelons qu'en 1907, la France se remet à peine de I'Affaire Dreyfus et que la famille Weil, côté maternel et juif de Proust, a toujours voulu s'assimiler - aussi aucun personnage ne rappelle-t-il son origine juive, alors trop délicate à assumer. II faudra attendre un an pour que naisse Swann dans le Cahier 4 et, avec lui, l'occasion d'aborder ce sujet de manière détournée car extérieure au cercle familial. Pour lors, le personnage de Swann n'est présent qu'à travers l'un de ses modèles réels, le grand-oncle de Proust, Louis Weil (1815-1896). Connu pour ses aventures amoureuses, ce grandoncle prêtera à Swann son goût pour les actrices, ainsi qu'au personnage de l'oncle Adolphe. Cependant, dans le manuscrit intitulé « Une soirée à la campagne », Proust masque la judéité de sa famille, et donc celle du grand-oncle; autrement dit, même le modèle de Swann n'est pas encore juif. Comme Nathalie Mauriac-Dyer l'écrit dans sa Notice, « pour faire mémoire d'un oncle coureur sans mentionner sa judéité (ou pour parler de la judéité sans dire qu'elle se rattache à sa propre 
famille), il convient donc de poser sur son visage le masque d'un personnage de roman » $(S F, 219)$, ce qu'il fera l'année suivante en créant le personnage de Swann.

Quant à l'homosexualité, le silence de Proust est éloquent. Et pourtant, c'est en 1908 qu'il prévoit d'écrire un essai sur « la Race des Tantes - ou des Maudits » ${ }^{7}$. Il faut croire qu'en 1907, Proust n'a pas encore trouvé le moyen d'évoquer ce sujet tout en préservant sa vie privée; on sait qu'il se montre très prudent, allant jusqu'à se battre en duel, en 1897, contre Jean Lorrain qui, dans sa critique des Plaisirs et les Jours, a fait allusion à son homosexualité. Nathalie Mauriac-Dyer reconnaît elle-même que les quelques allusions à l'homosexualité, dans "Le côté de Villebon et le côté de Meséglise », ne permettent pas de conclure à un véritable traitement de ce sujet. Dans ce manuscrit, le narrateur songe à Louis II de Bavière et à ses " étranges amours » $(S F, 100)$, il évoque son " mécanicien » $(S F, 153)$, à savoir Alfred Agostinelli, son chauffeur et amant, et fait référence à quelques excursions qu'ils firent ensemble en Normandie en 1907. Les allusions sont sibyllines, comme l'évocation de Bretteville-l'Orgueilleuse, commune normande

$7 \mathrm{M}$. Proust, À La recherche du temps perdu, J.-Y. Tadié (dir.), Paris, Gallimard, 1988, vol. 3, p. 924-928, 930-933. 
où Proust et Agostinelli sont allés en octobre 1907, et réutilisée dans le nom d'un personnage, M. de Bretteville qui, lui, est le futur Charles Swann. Autant dire que ces masques destinés à cacher son homosexualité équivalent à un silence, car ils sont destinés à un public très restreint de lecteurs avertis. Déjà en 1896, Proust écrivait à Reynaldo Hahn, son grand amour à l'époque où il écrit Jean Santeuil: "Je veux que vous y soyez tout le temps mais comme un dieu déguisé qu'aucun mortel ne reconnaît " ${ }^{8}$. De même, dans Les Soixantequinze Feuillets, aucun lecteur ne peut déceler I'homosexualité du narrateur sous le masque des allusions.

À l'inverse de ces non-dits, Les Soixante-quinze Feuillets présentent une réalité brute, celle des deux femmes qui entourent Proust : sa mère et sa grand-mère. II est tentant de penser que toutes deux n'ont pas été masquées. Dans La Recherche, les deux personnages empruntent la plupart de leurs traits à ces deux modèles transparents, mais la mère est appelée « Maman » et la grand-mère "Bathilde», nom universel dans le premier cas, et fictif dans le second. Dans Les Soixante-quinze Feuillets, le narrateur ne se donne pas cette peine:

8 M. Proust, Correspondance, P. Kolb (éd. critique), Paris, Plon, 1976, vol. 2, p. 52. 
la mère s'appelle Jeanne $(S F, 34)$ et la grand-mère Adèle $(S F, 34)$, prénoms réels de Jeanne Weil (1849-1905) et Adèle Berncastell (1824-1890). Ces modèles sont certes évidents, mais Proust ne les avait jamais révélés ailleurs, ce qui prouve le caractère autobiographique des Soixante-quinze Feuillets. De même, Proust utilise plusieurs fois son prénom, Marcel (il le fera dans La Recherche, par accident), il se donne un frère $(S F, 48)$ dans lequel on reconnaît Robert Proust, et il nomme la domestique de la famille Félicie $(S F, 59)$, du nom de Félicie Fitau, la femme de chambre de Madame Proust - ce n'est pas encore Françoise, composée de plusieurs modèles réels dont Félicie Fitau et Céleste Albaret. La composition des personnages n'est donc pas achevée et le travail du pseudonyme est quasiment nul.

Le cas de la grand-mère va cependant ouvrir la voie au travestissement de la réalité. Dans "Une soirée à la campagne », elle est d'abord appelée Adèle, puis Cécile et enfin Octavie : Proust tâtonne dans son récit. Par ailleurs, le portrait de la grand-mère comporte sa part de ridicule; pour ne pas risquer d'entacher son souvenir, Proust en fait alors une tante $(S F, 27,29)$ avant de la faire redevenir grand-mère, et ce afin de brouiller les pistes autobiographiques. Le procédé étant imparfait (la superposition de masques ne suffit 
pas à préserver Adèle Berncastell du ridicule puisqu'elle reste reconnaissable), il finira par attribuer ses défauts à d'autres personnages, ou par les supprimer. Les deux sœurs de la grandmère, dans Du côté de chez Swann, hériteront des allusions obscures dont Adèle Berncastell était coutumière; quant au refus de se changer et à la saleté qu'évoque Proust dans Les Soixante-quinze Feuillets, il les fera disparaître dans La Recherche. De la même manière, Les Soixante-quinze Feuillets rapportent une réalité autobiographique, la mort de Jeanne Weil, décédée des suites d'une crise d'urémie en 1905 ; dans La Recherche, Proust va reporter cet épisode sur la grand-mère afin de préserver l'image de « pureté adorable, rayonnant d'une intelligence, d'une douceur enjouée que la douleur n'a pu jamais éteindre, une innocente gaieté » $(S F, 41)$ qu'il souhaite conserver chez sa mère. Dans le premier fragment toujours, cet hommage est contredit par la description de la crise d'urémie qui amoindrit la dignité de sa mère : visage rouge, yeux fatigués, embonpoint, difficulté à marcher. Plutôt que de s'y résoudre, Proust masque cette agonie en la transférant dans celle de la grand-mère du narrateur dans Le Côté de Guermantes; exemple précoce de la composition des personnages telle que Proust l'appliquera dans La Recherche, c'est-à-dire la réunion de 
plusieurs modèles réels dans un seul personnage. C'est ce qu'il confirme à Jacques de Lacretelle dans une lettre en 1918: « II n'y a pas de clefs pour les personnages de ce livre; ou bien il y en a huit ou dix pour un seul ${ }^{9}$.

Le personnage de la grand-mère offre un autre intérêt dans le même fragment des Soixante-quinze Feuillets: le narrateur y raconte avec beaucoup d'humour l'art de l'allusion qu'elle employait dans ses lettres.

Comme elle était spirituelle, lettrée, et trouvait que par prudence il ne faut jamais mettre de noms propres dans les lettres elle ne parlait de tout que par allusions, figures, énigmes, personne ne comprenait de qui elle voulait parler. $(S F, 28)$

Comme un exégète cherchant à élucider les modèles d'un roman à clefs, Proust dit « à vingt ans de distance [avoir] pu arriver à presque tout reconstituer » $(S F, 28)$.

Ma fille

Tiré hier Durandal Hollandais volant avec Je vous ennuie Madame. Ah! La folle, la folle, la folle. Nous avons été interrompus par vilain doc et ma mère vous êtes la reine du bal. Il a décrété que les enfants étaient anémiques. Ce Machut. Je l'ai regardé de nos quarante siècles mais vous auriez su mieux que moi ce qu'il fallait répondre, hélas je suis à Etampes. Je vous envoie deux ou trois Pends-toi Sévigné qui valent leur pesant d'or. Avez-vous reçu les hirondelles de Myroti. $(S F, 28)$

9 M. Proust, Correspondance, op. cit., 1989, vol. 17, p. 193. 
À partir de ce message codé, Proust se livre à une véritable explication de texte. Pour ne citer que quelques clefs qui concernent la première phrase, «Tirer Durandal » signifie se mettre en colère, en référence à l'épée de Roland dans La Chanson de Roland ; le « Hollandais volant » est le surnom d'un banquier hollandais que la famille Proust croyait voleur ; " Je vous ennuie Madame » désigne une femme qui dérangeait Adèle Berncastell à chaque fois qu'elle la rencontrait ; la première phrase pouvant ainsi être traduite par « J'ai défendu hier notre banquier hollandais contre cette femme assommante ». Concernant l'art du pseudonyme, nous pouvons donc penser que Proust a été à bonne école auprès de sa grand-mère. Notons que le Contre Sainte-Beuve montre également cet art de l'allusion et de la citation, cette fois entre le narrateur et sa mère. La "Conversation avec Maman » en donne un exemple:

- Maman.

- Tu m'avais bien appelée, mon chéri ?

- Oui.

- Je te dirai que j'avais peur de m'être trompée et que mon Loup me dise :

C'est vous Esther qui sans être attendue...

Sans mon ordre on porte ici ses pas.

Quel mortel insolent vient chercher le trépas.

- Mais non, ma petite Maman.

Que craignez-vous, suis-je pas votre frère?

Est-ce pour vous qu'on fit un ordre si sévère? 
- Cela n'empêche pas que je crois que, si je l'avais réveillé, je ne sais pas si mon Loup m'aurait si béatement tendu son sceptre d'or. ${ }^{10}$

Dans cet extrait, mère et fils échangent des citations de l'Esther de Racine, le « sceptre d'or » étant pour finir une allusion à la clémence du roi Assuérus envers Esther, et donc à l'humeur de Marcel si sa mère l'avait réveillé. Simple habitude ou tradition familiale, Proust semble avoir intégré à son travail des pseudonymes ce jeu d'énigmes et de sous-entendus. Nathalie Mauriac-Dyer le dit elle-même : « il pratiquera lui-même volontiers, avec discrétion, cet art du travestissement et du cryptage dans son roman où, à travers un détail, le parent, l'ami, I'amant ou l'ennemi "reconnaissent" un sens qui échappe à la plupart » $(S F, 233)$.

\section{Le masque comme révélateur}

Passons à présent de l'art du pseudonyme à l'art du nom. À la recherche du temps perdu n'est pas un roman à clefs; Proust l'a suffisamment nié et Les Soixante-quinze feuillets nous le montrent bien car, en poursuivant la rêverie poétique sur le nom qui avait commencé dans Jean Santeuil, Proust ajoute une valeur littéraire au pseudonyme : les noms inventés sont moins des masques

10 M. Proust, Contre Sainte-Beuve, B. de Fallois (éd. critique), Paris, Gallimard, 1954, p. 119-120. 
destinés à protéger la vie privée que des moteurs pour l'imagination et donc pour la création.

Cette évolution du masque et de ses fonctions est particulièrement visible en ce qui concerne la géographie. Dans le deuxième fragment, intitulé "Le côté de Villebon et le côté de Meséglise », se fait jour la genèse de Combray. En 1907, Combray n'existe pas encore - ce n'est qu'en 1909 que Proust réunira la maison d'Auteuil et celle d'illiers pour en faire la maison de la tante Léonie - toujours dans cette perspective d'assemblage de réalités autobiographiques. Néanmoins, la géographie réelle du pays d'Illiers, future Illiers-Combray, est reconnaissable dans ce fragment à travers les toponymes suivants :

Je fus étonné d'apprendre par mon mécanicien qu'en prenant à droite de Chartres la route de Nogent-le-Rotrou puis en tournant deux ou trois fois à gauche on arrive au château de Villebon. $(S F, 53)$

Villebon se situant au nord d'Illiers, Chartres au nord-est et Nogent-le-Rotrou à l'ouest, les indications du narrateur sont réalistes. Le commentaire qui suit oriente toutefois cette toponymie vers le rêve : "C'est pour moi comme si on me disait qu'après avoir pris un premier chemin et un second chemin on arrive au pays des rêves » $(S F, 53)$. Et le narrateur insiste sur le caractère imaginaire de ces lieux qui, pourtant, existent : «Villebon était aussi 
lointain, aussi abstrait que le Nord ou l'Espagne », «Pendant bien longtemps je ne connus Villebon que par ces mots: "la route de Villebon, aller du côté de Villebon" » $(S F, 59)$. Autrement dit, Proust ne connaît le château de Villebon que par son nom, nom qui lui fait découvrir les pouvoirs de l'imagination associée à un simple toponyme. À partir de ce constat, pourquoi ne pas changer les noms pour les adapter à son imagination ? Le récit bascule alors du réel à la fiction. C'est pourquoi Proust, dans le même fragment, change la route de Bonneval - réelle, au sud d'Illiers - en " côté de Meséglise » $(S F, 57)$, nom fictif inspiré de «Méréglise », ville réelle située à l'ouest : la topographie est alors bouleversée et la toponymie inventée, même si «le côté de Villebon » ne sera remplacé par « le côté de Guermantes » que plus tard.

En même temps qu'il prend des libertés avec la géographie, Proust élabore sa poétique du nom. Le narrateur commence par déplorer la difficulté « d'épuiser la particularité d'un pays » par le langage, le «malaise intellectuel » face à «l'inexprimable » $(S F, 62)$, et trouve finalement la solution dans le nom poétique, seul capable d'exprimer «l'essence mystérieuse » $(S F, 62)$ des lieux. C'est ici que le masque du nom dépasse le simple pseudonyme et devient un moteur pour l'imagination, 
une création poétique à part entière, porteuse de vérité. Le nom inventé n'est plus un voile pudique, mais un révélateur. Proust explique ainsi le cheminement de sa pensée :

Même aujourd'hui où tous les lieux de la terre m'ont l'un après l'autre refusé l'essence mystérieuse que je rêvais en chacun d'eux et m'ont appris l'un après l'autre l'inutilité de ces voyages dont leur nom éveillait en moi un désir insensé, il me semble que cette avenue doit contenir réellement quelque chose d'analogue à ce que j'ai tant rêvé. [...] Je sens, et cette fois non plus par l'imagination, je sens directement l'essence mystérieuse d'un pays, c'est mon imagination qui voit, c'est mon rêve que je vais vivre, mais alors je m'éveille. [...] Car il est vraiment des choses qui ne doivent point nous être montrées. $(S F, 63)$

Ce dont parle Proust, c'est l'expérience décevante du voyage, à laquelle il préfère l'expérience poétique du nom, plus évocateur et plus vrai. C'est ce que nous apprend également le manuscrit sur les "Noms nobles », où Proust montre la supériorité du Nom sur

le langage de la poésie [parfois] trop précis encore, trop chargé de mots et par conséquent d'images connues, pour ne pas troubler ce courant mystérieux que le Nom, cette chose antérieure à la connaissance, fait courir, semblable à rien que nous connaissions comme parfois dans nos rêves [...]. (SF, 95)

Pour le dire autrement, le toponyme fictif, le pseudonyme, est un masque plus vrai que la réalité. Nous reconnaissons ici, déjà bien affirmée, la théorie onomastique qu'exposera le narrateur de La Recherche dans Nom de pays: le nom. 
Justement, les masques que pose progressivement Proust sur sa réalité autobiographique prennent une signification déjà poétique dans Les Soixante-quinze Feuillets - c'est ce qui rend ces manuscrits si émouvants, les balbutiements de La Recherche y côtoyant les théories les plus accomplies. Derrière chaque nom fictif, I'on devine la rêverie du narrateur, la poésie future du pays de Combray (qui n'est pas encore Combray), de Balbec (qui n'est pas encore Balbec mais " C. ", initiale de Cabourg où Proust allait effectivement en vacances), de Venise (sujet du sixième et dernier manuscrit), et bien sûr la fascination pour les familles nobles et pour les châteaux. Dans ce dernier cas, l'accusation de snobisme qui est faite contre Proust est à nouveau battue en brèche, à une époque où, pourtant, Proust fréquente encore les salons mondains. Selon lui, «il ne peut y avoir identité entre la poésie inconnue qu'il y a dans un nom c'est-à-dire une urne d'inconnaissable et les choses que l'expérience nous montre et qui correspondent à des mots, aux choses connues » $(S F, 97)$. II faut entendre ici la déception du narrateur face aux nobles si différents de ce que leurs noms laissaient présager. En témoigne, dans le manuscrit sur les «Noms nobles », ce seigneur allemand dont le nom est " traversé comme d'un souffle de poésie fantastique » $(S F, 96)$, 
cet autre dont le nom est celui « d'un ruisseau né dans la Forêt-Noire au pied de l'antique Wartbourg et traverse toutes les vallées hantées des gnomes » $(S F, 96)$. Le narrateur rencontre ces deux seigneurs et fait part de sa désillusion : ces princes sont communs et dénués de poésie. La conclusion est sans appel : "la réalité [du nom] est bien plus vivace que l'autre » $(S F, 98)$. Dans ce cas précis, c'est la personne qui est un masque pour le nom ; c'est le réel qui masque la vérité. Le narrateur vivra à nouveau ce type de désillusions dans La Recherche, quand la vulgarité ou la bêtise de certains personnages le heurteront après qu'il leur aura prêté de grandes qualités à partir de leur patronyme. Reviendra également cette idée que le nom, le masque, est parfois plus vrai que la réalité - c'est le cas pour les personnages multiples tels que Charlus ou Albertine, dont la superposition de masques finit par définir la personnalité. Chez Proust, parfois, le masque est la vérité.

Les Soixante-quinze Feuillets présentent l'évolution de Proust dans son rapport à l'autobiographie : on y décèle la réalité, à peine masquée voire affichée, derrière la fiction, on y devine tel ou tel élément de $\mathrm{La}$ Recherche dont on ignorait l'origine. La valeur génétique de ces manuscrits est évidente, mais c'est peut-être en matière de poétique que ce document est inestimable, car 
cette étape entre Jean Santeuil et les Cahiers qui vont déboucher sur La Recherche est fondamentale. Dans ces Soixante-quinze Feuillets, Proust élabore sa théorie du Nom qui va l'aider à passer de l'autobiographie à la fiction et à se servir des masques romanesques non plus pour cacher mais pour révéler - et l'on sait combien Proust concevait son œuvre comme une révélation. Le chemin n'est pas achevé, puisque l'écrivain va passer par le Contre Sainte-Beuve avant de commencer véritablement La Recherche, mais le détour par l'essai ne sera au fond qu'un masque supplémentaire appliqué à la réalité afin de se libérer des contraintes que le réel lui imposait : la crainte de froisser sa famille, la crainte des exégètes et des biographes. Aussi veut-on bien le croire lorsqu'il écrit, malgré I'autobiographie manifeste de Jean Santeuil et des Soixante-quinze Feuillets, malgré la tendance à voir La Recherche comme un roman à clefs : « Tout est fictif, laborieusement car je n'ai pas d'imagination [...] " ${ }^{11}$. C'est ce «labeur » que nous montrent Les Soixante-quinze Feuillets à travers le travail du nom, du masque et de la composition des personnages qui va donner naissance, cinq ans plus tard, à l'univers romanesque de La Recherche.

Date de réception de l'article: 27.08.2021 Date d'acceptation de l'article: 20.09.2021

11 N. Mauriac-Dyer, [cité d'après :] M. Proust, Les Soixantequinze Feuillets et autres manuscrits inédits, op. cit., p. 204. 


\section{bibliographie}

Proust M., À La recherche du temps perdu, J.-Y. Tadié (dir.), Paris, Gallimard, 1988, vol. 3.

Proust M., Contre Sainte-Beuve, B. de Fallois (éd. critique), Paris, Gallimard, 1954.

Proust M., Correspondance, P. Kolb (éd. critique), Paris, Plon, 1976, vol. 2 ; 1989, vol. 17.

Proust M., Correspondance, J. Picon (éd. critique), Paris, Flammarion, 2007.

Proust M., Jean Santeuil, Paris, Gallimard, 2001.

Proust M., Les Soixante-quinze feuillets et autres manuscrits inédits, N. Mauriac-Dyer (éd. critique), Paris, Gallimard, 2021. Tadié J.-Y., Proust et le roman, Paris, Gallimard, 1971. 


\section{abstract}

\section{Les Soixante-quinze Feuillets by Marcel Proust or the production of the mask}

In Search of Lost Time, the narrator is not the writer, and we can find out several models behind one character. In other words, Proust created a mask he put on reality to avoid criticism and problems with his family, but also to look for the essence of beings and things by focusing the truth. Pseudonyms are not a game but a real poetic of the Name. The recent publication of his Soixante-quinze Feuillets (Gallimard, 2021) shows how Proust transformed his autobiography into a fictional world. That preliminary version exposes the methods he used to hide himself and the way he was experimenting the powers of the imagination and composition. It is the writer's laboratory, an important source for genetic study of Proust's masterpiece.

\section{keywords}

laboratory, genetic, autobiography, pseudonym, poetic

\section{mots-clés}

laboratoire, génétique, autobiographie, pseudonyme, poétique 


\section{anne-aël ropars}

Anne-Aël Ropars est agrégée de Lettres modernes. Elle prépare actuellement un Doctorat en Littérature Française Poétique de Jean Giono par lui-même - dans le cadre d'un contrat doctoral à l'Université de Bretagne Occidentale sous la direction du Professeur Sophie Guermès. Son domaine de recherche est la littérature française du XXe siècle, notamment les œuvres de Marcel Proust et de Jean Giono sur lesquels elle a publié plusieurs articles. Chargée de cours à l'université Victor Segalen de Brest, elle est membre du Centre d'Étude des Correspondances et Journaux Intimes de I'UBO et participe au comité de lecture de la revue TransversALL.

ORCID : 0000-0003-4998-7304 